\title{
Fecundidade e participação no mercado de trabalho brasileiro
}

Fertility and participation in the Brazilian labor market

\author{
Marina Silva Cunha \\ Universidade Estadual de Maringá \\ Marcos Roberto Vasconcelos \\ Universidade Estadual de Maringá
}

\section{Abstract}

This paper analyzes the determinants of fertility and participation of females in Brazil's labor market. For such a purpose, we used the information from the national home sampling survey of IBGE, from 1995 to 2009, and the bivariate probit model. As expected, decreases in fertility were observed, as well as increased participation in the analyzed period. A negative effect of wages on the probability of fertility was also observed and a positive effect on the participation probability in the labor market. In addition, other control variables that affect the decisions of women were found including personal characteristics, condition in the family and place of residence. Furthermore, it was found that the effect of wages on fertility is higher in the lower tail of the distribution of household income, and that the inverse occurs in participation of women in the labor market.

\section{Keywords}

female participation; fertility; wages; income.

JEL Codes J13; J22; J30.

\section{Resumo}

Este trabalho analisa os determinantes da fecundidade e da participação das mulheres no mercado de trabalho brasileiro. São utilizadas as informações da Pesquisa Nacional por Amostra de Domicílio do IBGE, de 1995 até 2009, e o modelo probit bivariado. Conforme o esperado, observou-se queda da fecundidade e aumento da participação no período estudado, bem como efeito negativo dos salários na probabilidade de fecundidade e positivo na probabilidade de participação no mercado de trabalho. Além disso, outras variáveis de controle incluidas relacionadas às características pessoais, condição na família e local de residência também afetam a fecundidade e a inserção das mulheres. Ademais, verificou-se que, na cauda inferior da distribuição da renda domiciliar, o efeito dos salários na fecundidade é maior, mas, no caso do engajamento no mercado de trabalho, é menor.

\section{Palavras-chave}

participação da mulher; fecundidade; salários; renda.

Códigos JEL J13; J22; J30. 


\section{Introdução}

Para a teoria econômica, a oferta de trabalho depende do seu custo de oportunidade, e, para as mulheres, esse custo está relacionado à maternidade (Becker, 1965). De fato, existe simultaneidade nos eventos ter filhos e engajamento no mercado de trabalho, o que dificulta o estudo do tema, decorrente do problema da endogeneidade, já que tanto o número de filhos pode explicar a oferta de trabalho quanto a oferta de trabalho se constitui em um importante determinante da fecundidade delas (Pazello; Fernandes, 2004). ${ }^{1}$

Conforme Browning (1992), há duas abordagens empíricas em economia para estimar essa relação entre fecundidade e participação. A primeira, denominada de "purista", trata a endogeneidade da fecundidade na oferta de trabalho por meio de um sistema de equações simultâneas. Já a segunda, chamada de "padrão", inclui a fecundidade do lado direito na equação de oferta de trabalho ou de participação, em que alguns trabalhos utilizam instrumentos que buscam contornar o problema da endogeneidade.

$\mathrm{Na}$ literatura internacional, há estudos considerando ora uma abordagem, ora outra, embora no Brasil, até o momento, os trabalhos tenham utilizado apenas a segunda abordagem. Assim, apesar do interesse dos pesquisadores e de estudos buscando entender esse fenômeno, inexistem estudos com dados brasileiros que tratem em conjunto da participação da mulher no mercado de trabalho e sua fecundidade, considerando a abordagem purista, adequada para modelos que envolvem o problema da endogeneidade.

Segundo o relatório do Banco Mundial (2011), que trata da igualdade de gênero, elevação nos salários das mulheres e na renda familiar estaria afetando a decisão da mulher de trabalhar fora de casa. Mas, para o mesmo estudo, as mudanças na fecundidade e na participação da mulher no mercado de trabalho, além das melhorias em educação e na capacidade de decisão delas, são fatores que não estão apenas associados, mas que podem estar se reforçando mutuamente. Ademais, ao discutir o planejamento familiar no Brasil, Carvalho e Brito (2005, p. 366) afirmam que "[...] a variável população não é uma variável neutra no processo social. Sabe-se, perfeitamente, que as variáveis demográficas não são exclusivamente 1 A presença de endogeneidade em modelos de regressão produz estimativas inconsistentes, conforme Greene (2012). 
dependentes ou determinadas por processos macroeconômicos, mas também determinantes".

Neste contexto, o objetivo deste estudo é analisar simultaneamente a fecundidade e a participação no mercado de trabalho das mulheres no Brasil, usando a abordagem "purista", identificando seus principais determinantes. Emprega-se o modelo de escolha discreta bivariado, que permite considerar a correlação existente entre fecundidade e participação, com a estimação de equações com erros correlacionados. $\bigcirc$ trabalho utiliza informações da Pesquisa Nacional por Amostra de Domicílios (PNAD), de 1995 até 2009, o que permitiu também identificar efeitos coortes.

A redução da fecundidade e o aumento da participação da mulher no mercado de trabalho são fenômenos observados em diversos países, inclusive no Brasil. Segundo D’Addio e D’Ercole (2005), dentre os países da OCDE, a taxa de fecundidade total tem apresentado tendência decrescente, reduzindo de 2,7 filhos por mulher, em média, em 1970, para 1,6 filho, em 2002, quando apenas o México e a Turquia não apresentavam taxas abaixo de 2,1, nível que corresponde à reposição da população. Conforme relatório do Banco Mundial (2011), enquanto nos Estados Unidos o tempo para redução do número médio de filhos por mulher, de 6 para 3, foi maior do que em 100 anos, nos países mais pobres, ou de desenvolvimento mais recente, essa queda tem sido mais rápida. No Brasil, por exemplo, em 1940, a taxa de fecundidade correspondia a 6,16 filhos por mulher, e, em 2010, a 1,90 filho, conforme IBGE $(2003,2012) .{ }^{2}$ Obviamente, a velocidade das quedas nas taxas de fecundidade se mostra diferente, bem como o seu nível em cada localidade, o que pode ser explicado pelo ritmo e pelo estágio da transição demográfica em que se encontram, além do contexto histórico, conforme discutido por Brito (2007, 2007a, 2008).

Por sua vez, a participação da mulher no mercado de trabalho, em 2006, era em média de $60 \%$, nos países da OCDE. ${ }^{3}$ Considerando também os países de menor desenvolvimento, as mulheres em 2008 já representavam mais de $40 \%$ da força de trabalho no mundo (Banco Mundial, 2011). ${ }^{4}$ No

2 No Brasil, nos anos de 1950, 1960, 1970, 1980, 1991 e 2000, a taxa de fecundidade total era igual a 6,$21 ; 6,28 ; 5,76 ; 4,35,2,89$ e 2,38, respectivamente (IBGE, 2003).

3 Conforme OECD (2007), essa taxa era menor na Turquia (23,8\%), enquanto alcançava o maior patamar na Islândia $(81,6 \%)$.

4 A participação da mulher no mercado de trabalho é menor no Oriente Médio e no Norte da África, onde atinge 26\%, e maior no Leste Asiático e no Pacífico, com 64\%, segundo o Banco Mundial (2011). 
Brasil, a expansão da participação feminina no mercado de trabalho também segue a tendência internacional. Para Scorzafave e Menezes-Filho (2001), dentre os determinantes desse aumento, estaria o aumento do nível de escolaridade e da idade média das mulheres. Contudo, embora se observe tendência positiva na proporção de mulheres na população economicamente ativa, essa ainda se encontra em um patamar abaixo do verificado entre os homens. Em 2008, de acordo com Fontoura e Gonzales (2009), enquanto $57,6 \%$ das mulheres estavam trabalhando, ou à procura de emprego, $80,5 \%$ dos homens se encontravam nessa situação.

Com relação à importância do tema, ressalta-se que o melhor entendimento do comportamento da fecundidade e da participação da mulher no mercado de trabalho é de essencial importância para a tomada de decisão dos gestores públicos. Conforme Del Boca e Locatelli (2006), se inicialmente o aumento da participação da mulher no mercado de trabalho pode contribuir positivamente para a sustentabilidade do sistema previdenciário, aumentando o volume de contribuições, como no caso de alguns países europeus, posteriormente, a redução da população em idade ativa - em decorrência da menor fecundidade das mulheres que trabalham - pode ter consequências negativas para esse mesmo sistema e para o próprio crescimento econômico de longo prazo. Daí a preocupação de alguns países com a implementação de políticas públicas buscando reconciliar maternidade e carreira profissional, tais como maior flexibilidade no emprego (jornada de trabalho) e redução no custo de oportunidade potencial das crianças (oferta de benefícios para filhos, licença-maternidade e creches).

Tal preocupação pode parecer ainda distante dos formuladores de políticas públicas brasileiros, uma vez que o Brasil passa atualmente pela fase em que maior parcela de sua população está em idade economicamente ativa e, assim, capaz de ampliar a capacidade de produção nacional. No entanto, segundo IBGE (2013), mantidos os atuais parâmetros demográficos, com destaque para a tendência de queda de fecundidade, em três décadas começar-se-á a observar decréscimo da população brasileira.

A evolução da fecundidade também pode afetar o nível de desigualdade de um país e, portanto, seu desenvolvimento econômico. Conforme Brito (2008), a taxa de fecundidade é maior entre mulheres com menor nível de renda per capita, ou seja, a tendência é de que mulheres com mais filhos, ou famílias mais numerosas, no Brasil, estejam entre as mais pobres. Embora, para Leoni et al. (2010), a queda da fecundidade tenha reduzido a dificul- 
dade das mulheres para trabalhar, especialmente os cônjuges femininos, aumentando a renda familiar e diminuindo a pobreza. ${ }^{5}$

Na seção dois, faz-se breve revisão teórica e empírica sobre a abordagem econômica do tema. A metodologia empregada e uma descrição das variáveis utilizadas na pesquisa são expostas na seção três. Na seção seguinte, apresentam-se e discutem-se os resultados dos modelos estimados. Finalmente, a seção cinco traz as considerações finais.

\section{Teoria e evidências}

Até os anos 1950, a análise da fecundidade era um tema negligenciado pela teoria econômica tradicional em razão da dificuldade de incorporá-la ao arcabouço da teoria do consumidor (Willis, 1973). Além disso, diferentemente da previsão de Malthus, de que aumentos da renda proporcionariam elevação da fecundidade, o crescimento da renda familiar nos países desenvolvidos se associava à queda da fecundidade, afastando assim o risco da "explosão demográfica".

Tal situação se alterou com as contribuições de Gary Becker, que vieram a se constituir a base da literatura na área. Becker (1965) formulou uma teoria da alocação do tempo para as famílias, considerando várias atividades em que elas são produtoras e consumidoras. Nessa teoria, os filhos podem ser interpretados como um bem durável para as famílias, que os demanda e os produz (Becker, 1960).

No entanto, a realidade, em especial em países desenvolvidos como os Estados Unidos, apresentava alguns fatores que justificassem relação inversa à esperada pela teoria de Becker, tais como o declínio da mortalidade infantil, o aumento do conhecimento dos métodos contraceptivos, a redução da discriminação contra as mulheres e o aumento do custo das crianças. Dentre esses custos, que determinariam a qualidade das crianças, estariam incluídos os da educação, alimentação, saúde e do cuidado, geralmente da mãe. Dessa forma, os salários desempenhariam papel relevante na decisão de ter filhos. Além disso, considerando esse arcabouço, existiria

5 Ademais, os resultados do trabalho de Berquó e Cavenaghi (2006), que analisaram os dados da PNAD de 2004 e dos Censos de 1991 e 2000, apontam redução mais acentuada da fecundidade na área rural e nas Regiões Norte e Nordeste do país e sugerem que pode haver declínio nos segmentos menos favorecidos da população, que possuem o maior número médio de filhos. 
uma relação positiva entre renda e filhos, que teria intensidade menor na quantidade de filhos do que na sua qualidade.

Valendo-se da base teórica desenvolvida por Becker, de forma pioneira, Mincer (1963), abordou-se a simultaneidade entre os determinantes da fecundidade e do engajamento das mulheres na força de trabalho e discutiu-se a importância do custo de oportunidade ou do preço na função demanda por filhos. Os resultados indicaram um efeito preço negativo maior do que o efeito renda positivo, o que estaria de acordo com os fatos observados historicamente de redução da fecundidade e aumento da renda e o seu impacto esperado na demanda por filhos. Assim, não haveria causalidade entre as duas variáveis, mas seriam escolhas simultaneamente determinadas pelas mesmas variáveis econômicas, tais como o salário da mulher e o do marido.

Willis (1973), com base nessas contribuições pioneiras para a teoria econômica, fez uma formalização mais detalhada da maternidade e da oferta de trabalho, incorporando na função utilidade da família a satisfação derivada da qualidade e da quantidade de filhos, além daquela obtida das demais mercadorias.

Com relação às evidências, Moffit (1984) é um exemplo da aplicação da abordagem purista do tema, em que a participação e a fecundidade são tratadas conjuntamente. Ao analisar dados longitudinais para os Estados Unidos, de 1968 até 1975, de aproximadamente 1.500 mulheres casadas e com idade entre 14 e 24 anos, Moffit conseguiu captar o efeito do tempo do casamento na fecundidade, que foi positivo nos primeiros três anos e negativo nos anos seguintes, além do efeito negativo esperado dos salários na fecundidade e positivo na participação.

Outro trabalho a utilizar um sistema de equações simultâneas para o tema é o de Di Tommaso (1999), com foco no mercado de trabalho da Itália, nos anos 1987, 1989 e 1991. Os resultados desse trabalho indicaram o "salário" como a variável mais relevante na propensão a ter filhos e a participar do mercado de trabalho para a mulher, mas não confirmaram a tendência de queda na fecundidade e de aumento da participação nas coortes estudadas, uma vez que as estimativas indicaram propensão decrescente de participação das coortes mais antigas para as mais jovens e o contrário com a fecundidade. Buscando analisar conjuntamente a participação e a fecundidade, Di Tommaso e Weeks (2000) utilizaram os modelos bivariados e multinomial para variáveis dependentes discretas, mas concluíram que o último melhor se ajustou às informações analisadas. 
Del Boca e Locatelli (2006), em um trabalho de ampla revisão da literatura acerca do tema, chegaram à conclusão de que as evidências internacionais, geralmente, estão de acordo com o sugerido pela teoria, isto é, os salários têm efeitos positivos na participação feminina, espelhando um prêmio pelo trabalho, e negativo na fecundidade, refletindo o custo de oportunidade da maternidade.

Como indicado, no Brasil há somente trabalhos que seguem a abordagem padrão, em que a maternidade se destaca como variável explicativa para a inserção feminina no mercado de trabalho. Sedlacek e Santos (1991) analisaram a participação da mulher cônjuge nesse mercado, com base em informações da PNAD, para o período 1983 até 1988, e estimaram modelos probit, em que foi possível observar a importância da inclusão de características da família nas regressões. Por exemplo, a renda do marido apresentou efeito negativo na probabilidade de participação, da mesma forma que a presença de filhos menores, embora a presença de filhos mais velhos, com idade entre 10 e 17 anos de idade, não tenha tido efeitos significativos.

Scorzafave e Menezes-Filho (2001) abordaram diferentes aspectos da taxa de participação na força de trabalho da mulher. Inicialmente, analisam a evolução da participação feminina, de 1982 até 1997, em que constatam seu aumento, principalmente para aquelas com mais anos de estudo. Posteriormente, buscaram identificar os principais determinantes da participação no mercado de trabalho, em que se destacam, além daqueles associados ao capital humano, como escolaridade e idade, as mulheres cônjuges que também contribuíram positivamente para a maior inserção feminina. Observaram que, quanto maior o número de filhos mais novos, menor a probabilidade de participação da mulher e, por outro lado, o inverso ocorre para filhos com idade entre 11 e 17 anos.

Avelino e Menezes-Filho (2003) estimaram algumas especificações para a oferta de trabalho de mulheres e elasticidades em relação aos salários, com base em informações das PNADs para o período de 1992 até 1999. A maioria dos resultados encontrados mostrou um valor negativo na relação entre salários e oferta de trabalho, portanto inconsistente com a teoria econômica. Contudo, os efeitos negativos da presença de filhos na quantidade de horas ofertada pela mulher no mercado de trabalho foi de acordo com o esperado, ademais esse efeito foi mais evidente para filhos mais novos. 
No trabalho de Oliveira et al. (2009), que analisaram as diferenças de gênero no desemprego e inatividade, a variável "número de crianças no domicílio" foi a que mais se destacou, refletindo-se positivamente sobre o desemprego e a inatividade das mulheres, especialmente para aquelas mais pobres, o que para os autores estaria indicando diferenças de escolaridade e de acesso à creche. ${ }^{6}$

Para contornar o problema da endogeneidade da maternidade no engajamento da mulher no mercado de trabalho, Pazello e Fernandes (2004) utilizam como instrumento a presença de natimortos. Assim, comparam mulheres com filhos e sem, em que ambas tenham preferência por ter filhos e utilizam métodos de estimação tradicionais e o de pareamento. Os resultados desse trabalho indicaram efeito negativo da maternidade na participação da mulher no mercado de trabalho, que diminui no longo prazo. Além do mais, verificou-se que a jornada de trabalho das mulheres com filhos é menor, varia de acordo com o número de filhos e aumenta no longo prazo, e que, no longo prazo, o salário-hora das mulheres com filhos e sem eles não difere.

Ainda há na literatura nacional importantes trabalhos que abordam o tema sem, contudo, utilizar métodos de estimação para a participação ou oferta de trabalho, dos quais podem ser destacados Bruschini e Lombardi (1996), Bruschini (2000), Ramos e Soares (1995), Leoni (2000), Soares e Izaki (2002), Wajnman e Rios-Neto (2000) e Dias Junior (2010). Em geral, esses trabalhos analisam a maior participação feminina no mercado de trabalho e ressaltam mudanças no papel da mulher na família, especialmente a queda da fecundidade, e a sua responsabilidade doméstica e socializadora como inibidores dessa maior inserção.

Portanto, essa literatura, teórica e empírica, revela que, além dos salários, há outras variáveis importantes para o estudo da fecundidade e do engajamento da mulher no mercado de trabalho, tais como as características da família e a renda familiar. A seguir é apresentado o procedimento metodológico para analisar esse tema, considerando essas evidências, e também uma análise descritiva preliminar das variáveis utilizadas. Assim, são abordados os principais determinantes da participação e da fecundidade das mulheres, incorporando a simultaneidade dessa relação. 


\section{Metodologia}

\subsection{Material e métodos}

No presente trabalho, são utilizadas as informações das PNADs, de 1995 até 2009 , e consideram-se as mulheres de 18 até 60 anos de idade e aquelas que responderam adequadamente às perguntas sobre fecundidade e sobre as demais informações aqui utilizadas. Assim, após a eliminação das observações com alguma informação não declarada, chegou-se a 1.368 .318 mulheres nas PNADs. Para obter a expansão de cada observação da amostra, foi utilizada sempre a última versão dos pesos disponibilizados na pesquisa. Como até 2003 a PNAD não cobria a área rural da Região Norte, a fim de manter a homogeneidade nos dados, a partir desse ano, foram desconsiderados os dados referentes a essa região.

A probabilidade de participação no mercado de trabalho $\left(y_{1}^{*}\right)$ e a probabilidade de se ter filhos $\left(y_{2}^{*}\right)$ são as variáveis dependentes, e não são diretamente observadas. Com o intuito de captar a probabilidade de participação, construiu-se uma variável binária que assume valor igual a 1 , se a mulher trabalha ou está procurando trabalho, e o valor zero, se ela está fora do mercado de trabalho. A fecundidade é captada por uma variável binária que assume valor igual a 1, quando a mulher tem algum filho com idade menor que 15 anos. ${ }^{7}$ Para estimar essa relação, é utilizado um modelo probit bivariado, que permite estimar duas equações, com erros correlacionados, da mesma maneira que os modelos de regressão aparentemente não relacionados. Assim, as variáveis de interesse podem estar correlacionadas, como ocorre no caso da fecundidade e da participação, sem que haja problemas com as estimativas dos parâmetros. Uma especificação geral para esse modelo bivariado, considerando Greene (2012), pode ser descrita da seguinte forma:

$$
\begin{array}{ll}
y_{1}^{*}=\mathbf{x}_{1}^{\prime} \boldsymbol{\beta}_{1}+\varepsilon_{1 i} & y_{1}=1 \text { se, } y_{1}^{*}>0,0 \text { caso contrário } \\
y_{2}^{*}=\mathbf{x}_{2}^{\prime} \boldsymbol{\beta}_{2}+\varepsilon_{2 i} & y_{2}=1 \text { se, } y_{2}^{*}>0,0 \text { caso contrário }
\end{array}
$$

7 Nos resultados e nas discussões, também são incluídas estimativas com outras definições para essas variáveis, em que a fecundidade passa a ser indicada por uma variável binária que assume valor igual a 1 , se a mulher possui filho com idade igual a 2 anos ou menos, e a participação por uma binária que assume valor igual a 1 , se a mulher está ocupada com tempo integral. Para a construção da variável "fecundidade", foram utilizadas as informações referentes à condição do indivíduo na família, bem como sobre a idade do último filho nascido vivo. 


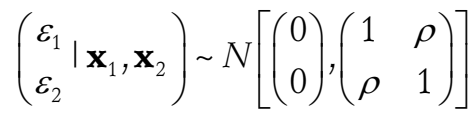

Conforme Greene (2012), há vários "efeitos parciais" que podem ser obtidos a partir das probabilidades bivariadas:

$$
\begin{aligned}
& \text { Prob }\left[y_{1}=1, y_{2}=1 \mid \mathbf{x}_{1}, \mathbf{x}_{2}\right], \\
& \text { Prob }\left[y_{1}=1, y_{2}=0 \mid \mathbf{x}_{1}, \mathbf{x}_{2}\right], \\
& \text { Prob }\left[y_{1}=0, y_{2}=1 \mid \mathbf{x}_{1}, \mathbf{x}_{2}\right] \mathrm{e} \\
& \text { Prob }\left[y_{1}=0, y_{2}=0 \mid \mathbf{x}_{1}, \mathbf{x}_{2}\right] .
\end{aligned}
$$

Desse modo, no primeiro caso, obtém-se o efeito de cada variável explicativa na probabilidade de ter filhos $(F)$ e de participar do mercado de trabalho $(\mathrm{P})$, ou seja, $(\mathrm{F} \cap \mathrm{P})$; no segundo, o efeito de cada variável na probabilidade de ter filho e não participar do mercado de trabalho ( $F \cap N P)$; o terceiro caso ocorre quando a mulher não tem filhos, mas participa do mercado de trabalho (NF $\cap \mathrm{P}$ ); e, por fim, tem-se o efeito de quando a mulher não tem filhos nem participa do mercado de trabalho $(\mathrm{NF} \cap \mathrm{NP}) .^{8}$ Também é calculado o efeito de cada variável na probabilidade não condicional de ter filhos ou de participar do mercado de trabalho. Esses efeitos marginais se constituem em estimativas do impacto na probabilidade de ocorrência da variável dependente de um aumento de uma unidade na variável explicativa.

Obviamente o salário da mulher está entre as variáveis explicativas, uma vez que se constitui em um importante determinante do custo de oportunidade de ter filhos e de participar do mercado de trabalho. Contudo, conforme mostraram Di Tommaso e Weeks (2000), a utilização dessa variável tem dois problemas: 1) o salário é observado apenas para as mulheres que estão trabalhando; e 2) pode estar correlacionado com fatores não observados que afetam a inserção econômica e a maternidade. Visando solucionar esses dois problemas, o presente trabalho segue a solução padrão adotada por Di Tommaso e Weeks (2000), utilizando os salários estimados em uma equação minceriana, pelo procedimento de Heckman (1979), para as mulheres que trabalham, corrigindo o viés 
de seleção. ${ }^{9} \mathrm{Na}$ equação de salários, é utilizado o logaritmo do salário hora como variável dependente e, dentre as variáveis explicativas, estão experiência - obtida subtraindo da idade, em anos, os anos de estudo e os seis anos que representam a idade pré-escolar - e seu quadrado, além de cinco variáveis binárias para representar seis níveis educacionais, em que os analfabetos estão no nível um, a categoria de referência, seguido pelo nível dois, com 1 até 3 anos de estudo; o nível três, com 4 a 7 anos de estudo; o nível quatro, com 8 a 10 anos de estudo; o nível cinco, com 11 a 14 anos de estudo, e o nível seis, com 15 anos de estudo ou mais. Além dessas variáveis, na equação de seleção são incluídas duas variáveis binárias: uma com valor igual a um quando a mulher é chefe da família; outra, com valor zero, quando a mulher é casada. Assim, com base nos parâmetros estimados por tal procedimento, capta-se o salário esperado para a oferta de trabalho das mulheres, o qual reflete basicamente seu investimento em capital humano.

Por sua vez, dentre as variáveis explicativas das equações de participação e de fecundidade estão, além do salário estimado (logaritmo/hora), a idade, em anos, e seu quadrado que buscam captar o comportamento da mulher ao longo do seu ciclo de vida; a renda domiciliar total como proxy de riqueza familiar; uma variável binária com valor igual a um quando a mulher é branca ou amarela, uma variável binária com valor igual a um quando é chefe da família e outra quando é casada; quatro binárias para distinguir as cinco grandes regiões, em que a Região Norte é a referência; uma variável binária que indica quando a residência é na área urbana e outra binária para distinguir as áreas metropolitanas das não metropolitanas. ${ }^{10}$

Buscando isolar as preferências quanto à participação e à fecundidade de diferentes gerações de mulheres, as informações de 1995 até 2009 foram empilhadas. Para tanto, são incluídas sete binárias, considerando as coortes de nascimento das mulheres, nas quais aquelas que nasceram após 1964 representam a categoria-base para as variáveis binárias que indicam as coortes de ano de nascimento. As coortes para os anos de nascimento buscam identificar as mudanças compartilhadas pela mesma geração ou

\section{Ver Mincer (1974).}

10 Por serem consideradas no trabalho, além das mulheres casadas, as solteiras, não foi incluído o salário do marido entre as variáveis explicativas, mas sim uma variável binária com valor igual a um quando eram casadas. Nos resultados e discussões são incluídas estimativas considerando parcelas específicas da amostra, tais como apenas as casadas ou as solteiras. 
pelos indivíduos que nasceram no mesmo período e captam diferenças de comportamento, hábitos, costumes e preferências. Além disso, incluiu-se uma variável binária para cada ano, buscando verificar se há mudanças estatisticamente significativas nos níveis de probabilidade de fecundidade e de participação no mercado trabalho ao longo do período estudado, considerando 1995 como ano-base. Portanto, além do efeito idade e coorte, é possível observar também o efeito período, que reflete mudanças conjunturais ou contemporâneas de determinada população, tais como econômicas e valores sociais.

Desse modo, uma contribuição deste artigo está na relevância de seu objetivo, que é o estudo da fecundidade e da participação da mulher no mercado de trabalho, além da metodologia utilizada que permite incorporar a simultaneidade dessa relação, possibilitando a realização de uma análise mais precisa do tema de interesse, de uma perspectiva ainda não abordada para o caso brasileiro.

\subsection{Análise descritiva}

A Tabela 1 traz a média das variáveis utilizadas no trabalho. Inicialmente, considerando a composição da população estimada, igual a 625.580.077, se nota que $49,75 \%$ das mulheres tinham pelo menos um filho menor que 15 anos. Com base nas informações das pesquisas nacionais por amostra de domicílios (PNADs), adicionalmente se verifica que, dentre essas mulheres, $12,60 \%$ tinham filhos com até dois anos de idade, e os restantes $37,15 \%$ tinham filhos com 3 até 14 anos de idade.

Com relação à participação, constata-se que 60,9\% das mulheres participavam do mercado de trabalho. Ademais, considerando as informações das PNADs, 7,4\% estavam à procura de emprego ou desempregadas, 19,1\% trabalhavam em tempo parcial e 34,4\% trabalhavam em tempo integral.

Os salários estimados, seguindo a metodologia apresentada anteriormente, são menores para as mulheres com filhos e para aquelas que não participam do mercado de trabalho. A renda domiciliar também possui comportamento semelhante, de acordo com esperado. ${ }^{11}$

11 Os valores monetários estão em real, de setembro de 2009; as informações para os demais anos foram atualizadas considerando o deflator para os dados das PNADs, disponibilizado no site do Instituto de Pesquisas Econômicas Aplicadas. Para mais detalhes, ver Corseuil e Foguel (2002). 
Tabela 1 Estatísticas descritivas, Brasil, 1995 - 2009

\begin{tabular}{|c|c|c|c|c|c|}
\hline \multirow[b]{2}{*}{ Variável } & \multirow[b]{2}{*}{ Total } & \multicolumn{2}{|c|}{ Fecundidade } & \multicolumn{2}{|c|}{ Participação } \\
\hline & & $\begin{array}{r}\text { Não tem } \\
\text { filhos }\end{array}$ & $\begin{array}{r}\text { Tem } \\
\text { filhos }\end{array}$ & $\begin{array}{r}\text { Não } \\
\text { participa }\end{array}$ & Participa \\
\hline Salário estimado & 2,58 & 2,64 & 2,52 & 2,43 & 2,68 \\
\hline Renda domiciliar & $2.277,81$ & $2.693,65$ & $1.857,77$ & $1.930,48$ & $2.500,04$ \\
\hline Anos de estudo & 7,59 & 8,04 & 7,15 & 6,16 & 8,51 \\
\hline Nível educacional 1 & 0,09 & 0,09 & 0,08 & 0,13 & 0,06 \\
\hline Nível educacional 2 & 0,11 & 0,10 & 0,12 & 0,14 & 0,08 \\
\hline Nível educacional 3 & 0,27 & 0,22 & 0,31 & 0,32 & 0,23 \\
\hline Nível educacional 4 & 0,17 & 0,16 & 0,18 & 0,17 & 0,17 \\
\hline Nível educacional 5 & 0,29 & 0,34 & 0,25 & 0,20 & 0,35 \\
\hline Nível educacional 6 & 0,08 & 0,10 & 0,06 & 0,03 & 0,11 \\
\hline Idade & 35,64 & 37,85 & 33,41 & 37,02 & 34,76 \\
\hline Branca & 0,55 & 0,59 & 0,52 & 0,54 & 0,56 \\
\hline Chefe & 0,23 & 0,22 & 0,25 & 0,17 & 0,27 \\
\hline Casada & 0,72 & 0,67 & 0,77 & 0,79 & 0,67 \\
\hline Norte & 0,06 & 0,05 & 0,06 & 0,06 & 0,05 \\
\hline Nordeste & 0,25 & 0,24 & 0,27 & 0,28 & 0,23 \\
\hline Sudeste & 0,47 & 0,50 & 0,44 & 0,45 & 0,48 \\
\hline Sul & 0,15 & 0,15 & 0,15 & 0,13 & 0,16 \\
\hline Centro-Oeste & 0,08 & 0,07 & 0,08 & 0,07 & 0,08 \\
\hline Região metropolitana & 0,35 & 0,38 & 0,33 & 0,32 & 0,37 \\
\hline Região urbana & 0,90 & 0,92 & 0,88 & 0,87 & 0,92 \\
\hline População estimada & 625.580 .077 & 314.363 .228 & 311.216 .849 & 244.087 .638 & 381.492 .439 \\
\hline
\end{tabular}

Fonte: Informações básicas das PNADs.

O nível de escolaridade é maior para aquelas que não têm filhos e que participam do mercado de trabalho. Por sua vez, a idade - que reflete as variações ao longo do ciclo de vida do indivíduo, das suas etapas de desenvolvimento, tanto biológicos e psicológicos quanto sociais e culturais - é maior para as que têm filhos e que não participam do mercado de trabalho.

É maior a proporção de mulheres da cor/raça branca dentre aquelas sem filhos e que estão no mercado de trabalho. As chefes de família não ultrapassam $30 \%$ do total de mulheres analisadas, e em torno de $70 \%$ possuem cônjuge, característica mais expressiva dentre as com filhos e as que não participam do mercado de trabalho. 
Com relação ao local do domicílio, verifica-se que a Região Sudeste é a que tem a maior proporção de indivíduos, seguida pelas Regiões Nordeste, Sul, Centro-Oeste e Norte, respectivamente. Ademais, aproximadamente $35 \%$ das mulheres residem em áreas metropolitanas e, em torno de $90 \%$ delas em domicílios urbanos.

A análise das informações das PNADs de vários anos permitiu distinguir os efeitos da idade, da coorte e do período, conforme salientado. $\mathrm{Ob}$ serva-se, na Figura 1, que a fecundidade e a participação no mercado de trabalho têm uma relação em forma de " $U$ " invertido com o ciclo de vida da mulher. No caso da fecundidade, ela atinge um máximo em torno de 35 anos, mas, para a participação no mercado de trabalho, essa relação não é tão evidente, já que a curva se apresenta mais achatada.

Pode-se observar ainda o comportamento da fecundidade e da participação no mercado de trabalho ao longo do ciclo de vida da mulher, considerando sua coorte, cujas mais recentes estão do lado esquerdo, e as precedentes, do lado direito. Ressalta-se que, nas três coortes mais jovens, há apenas mulheres da pesquisa de 2009 e que, nas três mais antigas, apenas da pesquisa de 1995. Portanto, neste trabalho há mulheres que nasceram em 1934, da pesquisa de 1995, até aquelas que nasceram em 1992, da pesquisa de 2009.

Para uma determinada idade, por exemplo, aos 27 anos, observa-se que os níveis de fecundidade entre as coortes mais recentes (coorte 85_89) são menores do que daquelas precedentes (coorte 70_74) e que ocorre o contrário em relação à participação, o que está de acordo com a queda da fecundidade e o aumento da participação no mercado de trabalho das mulheres, observado nas gerações mais novas.

Tanto a fecundidade quanto a participação também podem ser analisadas em cada décimo da distribuição da renda domiciliar, conforme a Figura 2, em que fica evidente que há menor participação e maior fecundidade entre as mais pobres, corroborando trabalhos anteriores. Verifica-se que, enquanto no primeiro décimo, com os menos favorecidos da população, em média a proporção de mulheres com filhos com idade inferior a 15 anos é de $65 \%$ e daquelas que participam do mercado de trabalho é de $43 \%$, por outro lado, no último décimo, esses valores se invertem e são, respectivamente, iguais a 34\% e 69\%.

Assim, verifica-se que há diferenças importantes na fecundidade e na participação entre os níveis de renda da população, ou seja, observa-se que não há homogeneidade em relação à fecundidade e à participação ao longo da distribuição da renda domiciliar. Para as demais variáveis analisadas, 
Figura 1 Proporção da população estimada com filhos menores que 15 anos e que participa do mercado de trabalho, por coorte, Brasil, 1995 - 2009
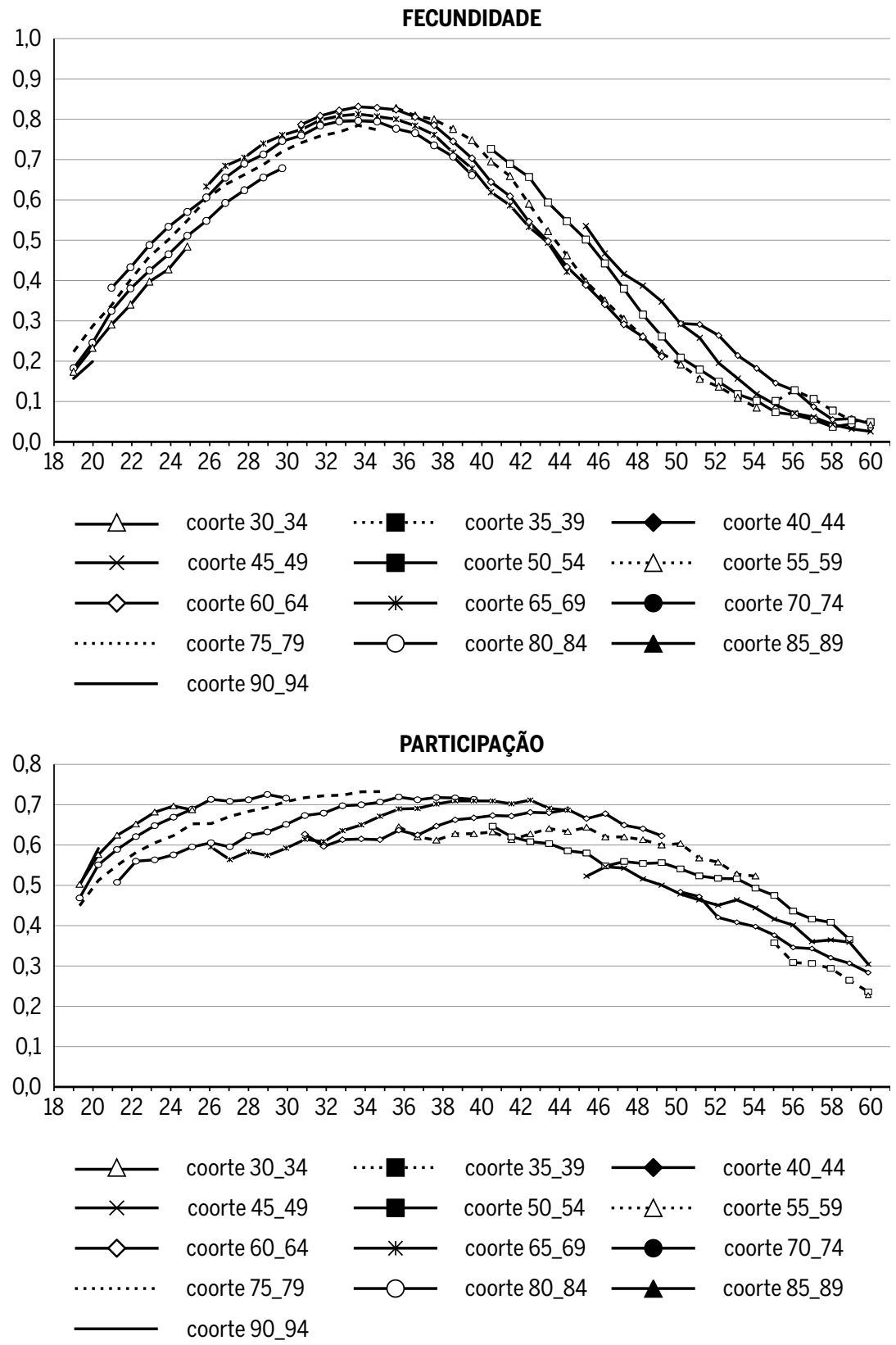

Fonte: Informações básicas das PNADs. 
também é possível notar que há outras diversidades, tanto em relação às características pessoais quanto regionais.

Figura 2 Média da fecundidade e da participação no mercado de trabalho, por décimo da distribuição do rendimento domiciliar, Brasil, 1995 - 2009

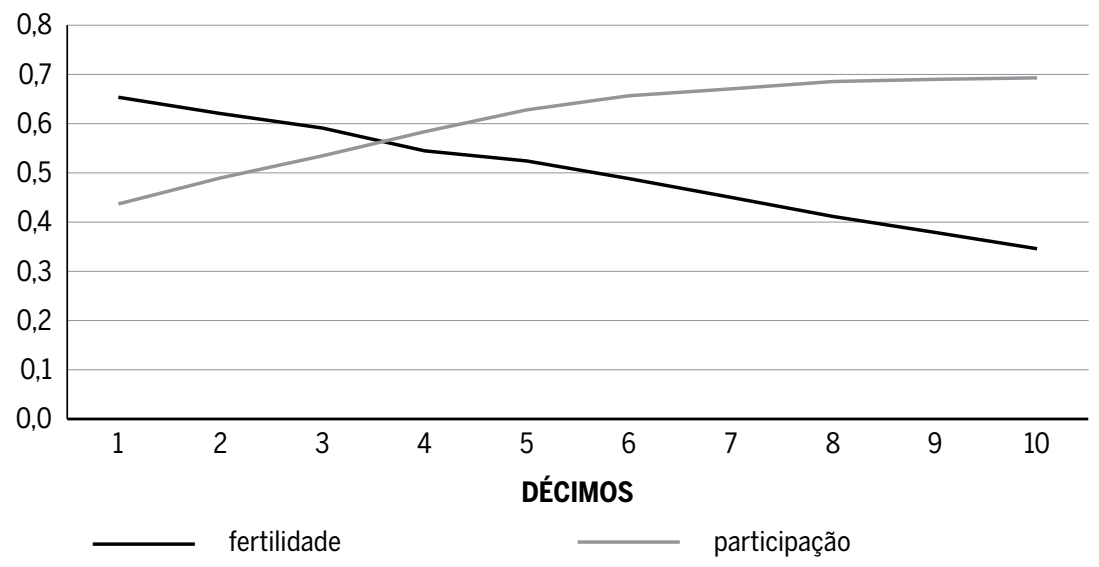

Fonte: Informações básicas das PNADs.

\section{Resultados e discussões}

As estimativas das equações de fecundidade e participação no mercado de trabalho estão na Tabela 2, cujos coeficientes foram estatisticamente significativos, considerando um nível de $5 \%$. Os Testes Wald para verificar se as duas equações são independentes também foram estatisticamente significativos, confirmando que o modelo bivariado é adequado para estudar esse tema. Inicialmente, o modelo foi estimado sem as variáveis de controle para as coortes de nascimento e período, mas na sequência são incluídas, buscando evidenciar seus efeitos.

Verifica-se que a correlação estimada entre as equações de fecundidade e de participação foi negativa. Isso indica que há efeitos não observados nas duas equações inversamente relacionados, como os levantados por Becker (1960), podendo-se citar, por exemplo, a redução da discriminação em relação às mulheres no mercado de trabalho e o avanço dos conhecimentos dos métodos contraceptivos. Conforme Mincer (1963), pode ser considerada a existência de dois argumentos para a relação negativa entre 
fecundidade e participação no mercado de trabalho: 1) a grande responsabilidade da mulher no cuidado com os filhos, que pode não permitir que ela entre ou volte para o mercado de trabalho; e, 2) o fato de aquelas que estão no mercado de trabalho poderem decidir restringir deliberadamente o tamanho de suas famílias.

Segundo Del Boca e Locatelli (2006), alguns estudos para os países nórdicos apontam a mudança dessa correlação entre a participação no mercado de trabalho e a fecundidade da mulher, de negativa para positiva. Possivelmente, a existência de políticas que buscam compatibilizar a maternidade e o trabalho das mulheres seria a principal responsável por tal mudança.

Os resultados para os efeitos dos salários na fecundidade e na participação no mercado de trabalho brasileiro estão de acordo com o esperado: na primeira, estimou-se efeito negativo, e, na segunda, positivo, ambos estatisticamente significativos. Portanto, o salário definido no mercado de trabalho, o qual reflete seu investimento em capital humano, representa uma variável relevante para a fecundidade e a inserção no mercado de trabalho. Conforme preconiza o modelo tradicional, a produção e o cuidado com os filhos são em tempo intensivo, e um aumento dos salários eleva o custo de oportunidade de se ter filhos, e o efeito substituição negativo, reduz a demanda por filhos. Assim, quanto maior o salário, maior o custo de oportunidade de ter filhos e também de não participar do mercado de trabalho.

Ressalta-se que os efeitos marginais, apresentados na Tabela A1, indicam que o "salário" é a variável mais importante na explicação da probabilidade de participação da mulher no mercado de trabalho. Já no caso da fecundidade representa o quarto determinante, após as variáveis "chefe", "casada" e "idade". Assim, verifica-se que a magnitude dos efeitos dos salários na participação da mulher no mercado de trabalho é maior do que o estimado para a equação de fecundidade. Esse menor impacto dos salários na probabilidade de fecundidade também foi observado no trabalho para a Itália realizado por Di Tommaso (1999).

No caso da renda domiciliar, estimou-se um sinal negativo para a probabilidade de se ter filhos e positivo para a probabilidade de participação no mercado de trabalho. Na equação de fecundidade, o sinal negativo indica que as mulheres mais ricas têm reduzido o número de filhos, o que pode ser explicado pelo maior investimento na sua qualidade, o que reflete no número de filhos, uma vez que esses custos são crescentes. Na equação de participação, o sinal positivo sugere que as mulheres na cauda superior da 
Tabela 2 Modelos estimados para as equações de fecundidade e de participação, Brasil, $1995-2009$

\begin{tabular}{|c|c|c|c|c|c|c|}
\hline \multirow[b]{2}{*}{ Descrição } & \multicolumn{2}{|c|}{ Modelo I } & \multicolumn{2}{|c|}{ Modelo II } & \multicolumn{2}{|c|}{ Modelo III } \\
\hline & $\begin{array}{l}\text { Fecun- } \\
\text { didade }\end{array}$ & $\begin{array}{r}\text { Partici- } \\
\text { pação }\end{array}$ & $\begin{array}{l}\text { Fecun- } \\
\text { didade }\end{array}$ & $\begin{array}{r}\text { Partici- } \\
\text { pação }\end{array}$ & $\begin{array}{l}\text { Fecun- } \\
\text { didade }\end{array}$ & $\begin{array}{r}\text { Partici- } \\
\text { pação } \\
\end{array}$ \\
\hline Intercepto & $-5,3043^{* *}$ & $-2,1696^{* *}$ & $-6,0338^{* *}$ & $-2,0377^{* *}$ & $-5,6285^{* *}$ & $-2,2954^{* *}$ \\
\hline Salário estimado & $-0,3742^{* *}$ & $0,5778^{* *}$ & $-0,3747^{* *}$ & $0,5777^{* *}$ & $-0,3835^{* *}$ & $0,5968^{* *}$ \\
\hline Idade & $0,3839 * *$ & $0,0778^{* *}$ & $0,4337^{* *}$ & $0,0659 * *$ & $0,4125^{* *}$ & $0,0775^{* *}$ \\
\hline $\begin{array}{l}\text { Idade ao } \\
\text { quadrado }\end{array}$ & $-0,0056^{* *}$ & $-0,0013^{* *}$ & $-0,0064^{* *}$ & $-0,0010^{* *}$ & $-0,0059 * *$ & $-0,0013^{* *}$ \\
\hline $\begin{array}{l}\text { Rendimento } \\
\text { domiciliar }\end{array}$ & $-2,6 \mathrm{E}-05^{* *}$ & $6,3 \mathrm{E}-06^{* *}$ & $-2,6 \mathrm{E}-05^{* *}$ & $6,7 \mathrm{E}-06^{* *}$ & $-2,6 \mathrm{E}-05^{* *}$ & $6,3 \mathrm{E}-06^{* *}$ \\
\hline Branca & $-0,0549 * *$ & $-0,1162^{* *}$ & $-0,0604^{* *}$ & $-0,1128^{* *}$ & $-0,0664^{* *}$ & $-0,1095^{* *}$ \\
\hline Chefe & $0,7796^{* *}$ & $0,2950^{* *}$ & $0,8002^{* *}$ & $0,2852^{* *}$ & $0,8114^{* *}$ & $0,2773^{* *}$ \\
\hline Casadas & $0,7478^{* *}$ & $-0,2795^{* *}$ & $0,7608^{* *}$ & $-0,2851^{* *}$ & $0,7636^{* *}$ & $-0,2856^{* *}$ \\
\hline Nordeste & $-0,1097^{* *}$ & $0,0157^{* *}$ & $-0,1124^{* *}$ & $0,0169 * *$ & $-0,1168^{* *}$ & $0,0210^{* *}$ \\
\hline Sudeste & $-0,1564^{* *}$ & $0,1351^{* *}$ & $-0,1569 * *$ & $0,1367^{* *}$ & $-0,1629 * *$ & $0,1429 * *$ \\
\hline Sul & $-0,0834^{* *}$ & $0,2631^{* *}$ & $-0,0806^{* *}$ & $0,2631^{* *}$ & $-0,0848^{* *}$ & $0,2685^{* *}$ \\
\hline Centro-Oeste & $-0,1241^{* *}$ & $0,1036^{* *}$ & $-0,1248^{* *}$ & $0,1033^{* *}$ & $-0,1299 * *$ & $0,1079 * *$ \\
\hline Área urbana & $-0,1364^{* *}$ & $0,1086^{* *}$ & $-0,1302^{* *}$ & $0,1041^{* *}$ & $-0,1174^{* *}$ & $0,0876^{* *}$ \\
\hline $\begin{array}{l}\text { Região } \\
\text { metropolitana }\end{array}$ & $-0,0551^{* *}$ & $0,0036^{* *}$ & $-0,0568^{* *}$ & $0,0046^{* *}$ & $-0,0592^{* *}$ & $0,0058^{* *}$ \\
\hline Coorte 30_34 & & & $1,7429 * *$ & $-0,5656^{* *}$ & $0,9201^{* *}$ & $-0,0289 * *$ \\
\hline Coorte 35_39 & & & $1,4188^{* *}$ & $-0,4992^{* *}$ & $0,6546^{* *}$ & $0,0438^{* *}$ \\
\hline Coorte 40_44 & & & $0,9034^{* *}$ & $-0,4091^{* *}$ & $0,2683^{* *}$ & $0,0578^{* *}$ \\
\hline Coorte 45_49 & & & $0,4847^{* *}$ & $-0,3295^{* *}$ & $-0,0073^{* *}$ & 0,0008 \\
\hline Coorte 50_54 & & & $0,1677^{* *}$ & $-0,2241^{* *}$ & $-0,1970 * *$ & $0,0195^{* *}$ \\
\hline Coorte 55_59 & & & $-0,0448^{* *}$ & $-0,1413^{* *}$ & $-0,2938^{* *}$ & $0,0270^{* *}$ \\
\hline Coorte 60_64 & & & $-0,0564^{* *}$ & $-0,0935^{* *}$ & $-0,2060 * *$ & $0,0132^{* *}$ \\
\hline Ano 1996 & & & & & $0,0487^{* *}$ & $-0,0698^{* *}$ \\
\hline Ano 1997 & & & & & $-0,0139 * *$ & $-0,0308^{* *}$ \\
\hline Ano 1998 & & & & & $-0,0140^{* *}$ & $-0,0497^{* *}$ \\
\hline Ano 1999 & & & & & $-0,0085^{* *}$ & $-0,0862^{* *}$ \\
\hline Ano 2000 & & & & & $-0,1151^{* *}$ & $0,0802^{* *}$ \\
\hline Ano 2001 & & & & & $-0,1702^{* *}$ & $0,1398^{* *}$ \\
\hline Ano 2002 & & & & & $-0,2166^{* *}$ & $0,1973^{* *}$ \\
\hline Ano 2003 & & & & & $-0,2292^{* *}$ & $0,2231^{* *}$ \\
\hline Ano 2004 & & & & & $-0,2399 * *$ & $0,2147^{* *}$ \\
\hline
\end{tabular}




\begin{tabular}{|c|c|c|c|c|c|c|}
\hline \multirow[b]{2}{*}{ Descrição } & \multicolumn{2}{|c|}{ Modelo I } & \multicolumn{2}{|c|}{ Modelo II } & \multicolumn{2}{|c|}{ Modelo III } \\
\hline & $\begin{array}{l}\text { Fecun- } \\
\text { didade }\end{array}$ & $\begin{array}{r}\text { Partici- } \\
\text { pação }\end{array}$ & $\begin{array}{l}\text { Fecun- } \\
\text { didade }\end{array}$ & $\begin{array}{r}\text { Partici- } \\
\text { pação }\end{array}$ & $\begin{array}{l}\text { Fecun- } \\
\text { didade }\end{array}$ & $\begin{array}{r}\text { Partici- } \\
\text { pação }\end{array}$ \\
\hline Ano 2005 & & & & & $-0,2335^{* *}$ & $0,1834^{* *}$ \\
\hline Ano 2006 & & & & & $-0,2492^{* *}$ & $0,1547^{* *}$ \\
\hline Ano 2008 & & & & & $-0,2951^{* *}$ & $0,1783^{* *}$ \\
\hline Ano 2009 & & & & & $-0,3571^{* *}$ & $0,2762^{* *}$ \\
\hline Rho & & $-0,1355539$ & & $-0,133182$ & & - 0,1257373 \\
\hline $\begin{array}{l}\text { Teste Wald para } \\
\text { rho }=0\end{array}$ & & $3,2 e+06^{* *}$ & & $3,1 \mathrm{e}+06^{* *}$ & & $2,8 \mathrm{e}+06^{* *}$ \\
\hline Log likelihood & & $-7,141 e+08$ & & $-7.108 e+08$ & & $-7,079 e+08$ \\
\hline
\end{tabular}

Fonte: Dados da pesquisa.

*Valores estatisticamente significativos com nível de 5\% e ${ }^{* *}$ de $1 \%$.

distribuição de renda podem ter maior suporte para conciliar a maternidade e a carreira profissional, tais como babás e creches.

Verifica-se que a idade em que a probabilidade de fecundidade estimada é máxima ocorre aos 34,3 anos e de participação aos 29,9 anos. Os resultados também indicam menor propensão das mulheres da cor/raça branca de fecundidade e de participação; já o contrário ocorre em relação à variável "chefe", que tem efeitos positivos nas duas equações, visto que o custo de oportunidade do chefe é maior, uma vez ser ele o maior provedor de recursos da família. Por seu turno, o fato de ser casada tem efeito positivo na fecundidade, mas negativo na participação, como o esperado. Esse fato pode ser explicado, de um lado, pelo modelo familiar ainda existente em que a mulher possui maior importância nas atividades domésticas apontado por Bruschini e Lombardi (1996), e de outro, o caráter secundário de sua inserção destacado em Soares e Izaki (2002).

Considerando as regiões, a chance de fecundidade é menor na Região Sudeste, em relação à Região Norte, tomada como categoria de referência, e a Região Sul tem a maior chance de participação. Os resultados também apontam que as residentes em áreas metropolitanas e urbanas têm menor probabilidade de fecundidade e maior de inserção econômica.

As estimativas do modelo, incluindo as variáveis binárias para diferenciar coortes, sugerem que a fecundidade é maior entre as coortes mais antigas e se reduz naquelas mais recentes, e, no caso da participação, as mais jovens têm maior probabilidade de estar no mercado de trabalho. Dentre os determinantes dessas transformações, estão a mudança na concepção 
de que os filhos são fonte de renda da família, a melhoria das condições de saúde, inclusive dos métodos contraceptivos, e a redução da discriminação de gênero. Assim, esses resultados corroboram o fato de que há mudança no comportamento das mulheres em relação à maternidade e à participação na atividade econômica. Para Ramos e Soares (1995), a remoção de barreiras culturais, religiosas e práticas discriminatórias foram fundamentais para a maior participação da mulher no mercado de trabalho brasileiro.

Conforme o esperado, os resultados estimados para as variáveis binárias - que diferenciam as observações do ano de 1995 dos demais - são estatisticamente significativos e indicam redução da fecundidade e aumento da participação no mercado de trabalho brasileiro, no período estudado. Desse modo, essas variáveis captaram essa tendência nacional, também observada no cenário internacional. Dentre os fatores que podem explicar essas mudanças, está o crescimento econômico, com a ampliação do mercado de trabalho. Outro fator que pode ter contribuído para a maior participação das mulheres seria o Programa Bolsa-Família, pois, conforme Tavares (2010), apesar do efeito renda gerado pelo programa, a redução da oferta de trabalho dos filhos, que devem permanecer na escola, propicia maior disponibilidade de tempo para as mães no mercado de trabalho. Ainda pode ser citada a relevância das políticas públicas em relação aos salários no país, em particular em relação ao salário mínimo, que apresentou crescimento real nesse período e tem reflexos importantes sobre os indivíduos que estão na cauda inferior da distribuição da renda e dos salários. ${ }^{12}$

Desse modo, após a inclusão das variáveis de controle de período, o efeito do ciclo de vida ainda aponta uma relação côncava, o efeito coorte sugere que as gerações mais jovens possuem probabilidade menor de terem filhos e, por fim, o efeito período indica redução da probabilidade de terem filhos ao longo do período analisado.

O efeito dos salários nas equações de fecundidade e de participação também foi obtido para cada décimo da distribuição da renda domiciliar, conforme a Figura 3, que traz os efeitos marginais. Verifica-se que, nos décimos inferiores, os efeitos dos salários na fecundidade são maiores, indicando a importância do custo de oportunidade do investimento em capital

12 De setembro de 1995 até setembro de 2009, o salário mínimo nominal aumentou de R\$ 100,00 para $R \$ 465,00$, e seu valor real correspondente, considerando como deflator o INPC com base na data inicial, foi igual a R \$100,00 e R\$255,93. Ver Neumark e Wascher (2008), para uma discussão dos efeitos do salário mínimo nas distribuições dos salários, dos rendimentos e da renda. 
humano das mulheres. É importante ressaltar que os salários expressam o nível de escolaridade das mulheres relevante nas escolhas no que diz respeito à maternidade.

Figura 3 Efeitos marginais em relação aos salários para as equações de fecundidade e de participação por décimos da distribuição da renda domiciliar, Brasil, 1995 - 2009
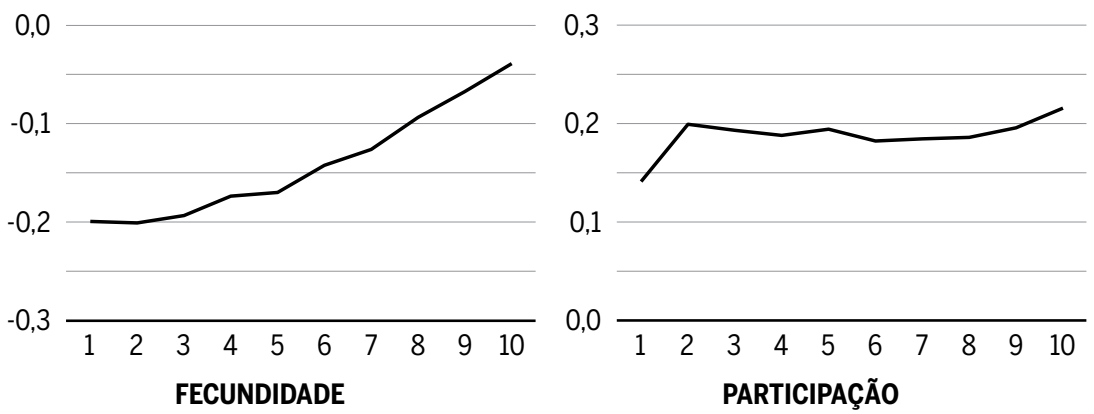

Fonte: Informações básicas das PNADs.

Por outro lado, os salários são mais importantes para aquelas mulheres que estão na cauda superior da distribuição da renda; no caso da inserção no mercado de trabalho, especialmente no último décimo, para os demais segmentos da população, o efeito não difere substancialmente. Assim, essa relação aponta que os salários das mulheres são relevantes para a fecundidade e o engajamento no mercado de trabalho e possui diferenças ao longo da distribuição da renda domiciliar.

As estimativas dos efeitos parciais das variáveis de controle, sobre a probabilidade das mulheres de fecundidade e de participação, estão apresentadas na Tabela A2. Nota-se que o impacto dos salários é positivo na probabilidade de as mulheres, com filhos ou sem, participarem do mercado de trabalho, e é negativo para a probabilidade de elas não participarem do mercado de trabalho.

Por fim, as estimativas foram obtidas para alguns grupos específicos de mulheres, conforme a Tabela 3, em que são apresentados os coeficientes estimados apenas para a variável "salário", embora os demais controles estejam incluídos. Inicialmente, observa-se que a correlação entre as duas equações é maior no ano de 1995, em relação ao de 2009, indicando que a conciliação entre maternidade e participação melhorou nesse período estudado. Ademais, nota-se redução dos efeitos dos salários na fecundidade 
e elevação na participação, refletindo o aumento da importância do capital humano na participação.

Quando as estimativas são obtidas apenas para aquelas casadas, os efeitos dos salários se modificam notadamente na equação de participação, apontando o custo de oportunidade delas entre se dedicar ao lar ou ao trabalho. Por outro lado, para as solteiras, a alteração mais expressiva ocorre na equação de fecundidade, em que o efeito negativo dos salários fica maior. Outra estimativa considerou mulheres de 18 até 49 anos. Nesse caso, o efeito dos salários fica mais forte nas duas equações, o que está de acordo com o período do ciclo de vida das mulheres em que a maternidade e a participação são maiores, conforme apontado na análise descritiva.

Tabela 3 Estimativas dos efeitos dos salários na fecundidade e na participação para diferentes grupos de mulheres, Brasil, 1995 - 2009 a

\begin{tabular}{lrrr}
\hline Descrição & Fecundidade & Participação & Rho \\
\hline Para o ano de 1995 ${ }^{\mathrm{b}}$ & $-0,37789^{* *}$ & $0,52774^{* *}$ & $-0,14589^{* *}$ \\
\hline Para o ano de 2009 b & $-0,33262^{* *}$ & $0,59289^{* *}$ & $-0,09522^{* *}$ \\
\hline Casadas & $-0,34161^{* *}$ & $0,61700^{* *}$ & $-0,14524^{* *}$ \\
\hline Solteiras & $-0,45167^{* *}$ & $0,54592^{* *}$ & $-0,05001^{* *}$ \\
\hline Com idade de 18 até 49 anos & $-0,42748^{* *}$ & $0,67673^{* *}$ & $-0,14955^{* *}$ \\
\hline $\begin{array}{l}\text { Fecundidade é igual a um, se tem filhos } \\
\text { com 2 anos ou menos }\end{array}$ & $-0,17961^{* *}$ & $0,59534^{* *}$ & $-0,24511^{* *}$ \\
\hline $\begin{array}{l}\text { Participação é igual a um, se está ocupada } \\
\text { em tempo integral }\end{array}$ & $-0,38578^{* *}$ & $0,32334^{* *}$ & $-0,14607^{* *}$ \\
\hline
\end{tabular}

Fonte: Informações básicas das PNADs.

aOs demais resultados dos modelos estimados para as outras variáveis de controle não estão apresentados. bEstimativa sem as variáveis de controle para coorte de nascimento e ano.

${ }^{*}$ Valores estatisticamente significativos com nível de $5 \%$ e ${ }^{* *}$ de $1 \%$.

Outra análise realizada foi a modificação da definição de fecundidade, considerando a existência de filhos com idade menor, ou seja, de dois anos ou menos. Observa-se que o efeito dos salários na probabilidade de ter filhos reduz-se quando a idade do filho é menor. Esse resultado indica que, quanto menor é o filho, maior seria seu custo de oportunidade, visto que o cuidado da mãe com o filho logo após o nascimento seria mais importante. Com isso, o impacto dos salários na probabilidade de ter filhos ficaria mais pronunciado em mulheres com filhos maiores. Note que esse resultado, além de mostrar que a correlação entre as duas equações é negativa, con- 
forme destacado, também sugere que quanto menor a idade do filho, mais forte é sua magnitude.

Por fim, o conceito de participação no mercado de trabalho também foi alterado, para considerar apenas aquelas mulheres com ocupação em tempo integral no mercado de trabalho, no qual se observam poucas alterações, em relação aos valores estimados na Tabela 2.

Assim, esses resultados mostram a relevância de analisar conjuntamente as decisões de participar e de maternidade, já que há correlação negativa e estatisticamente significativa entre essas variáveis, de modo que estimativas isoladas, por exemplo, da participação incluindo maternidade como controle pode levar a estimativas incorretas. Verifica-se a importância dos salários para a ampliação da oferta de trabalho no país e também de políticas que busquem flexibilizar o emprego, de forma que as mulheres possam participar em melhores condições do mercado de trabalho.

\section{Considerações finais}

Este trabalho mostrou diversos aspectos do comportamento da fecundidade e da participação no mercado de trabalho brasileiro da mulher. Inicialmente, os resultados obtidos estão de acordo com o que preconiza a teoria econômica e com as evidências para outros países de que há correlação entre fecundidade e participação, devendo ser estudadas simultaneamente, conforme realizado no presente estudo. As estimativas para o efeito dos salários na fecundidade é negativo e na participação é positivo, como o esperado.

Verificou-se que a correlação negativa entre participação e fecundidade é menor em 2009 do que em 1995, sugerindo que os efeitos entre essas variáveis estão menores. Nessa perspectiva, é possível dizer que, de um lado, as barreiras para o maior engajamento da mulher vêm diminuindo, embora ainda existam, e, de outro, que a possibilidade de ter o número de filhos desejado e no momento desejado está maior, o que, no caso brasileiro, se tem traduzido em menos filhos.

Observou-se o comportamento das mulheres em relação à fecundidade e à participação, considerando o período, sua coorte e seu ciclo de vida. Pôde-se constatar que, enquanto a fecundidade da mulher caiu, sua participação aumentou, de 1995 para 2009, ou seja, captou-se a tendência atual de queda na fecundidade e de aumento da participação das mulheres no mercado 
de trabalho brasileiro. Essa mudança também pôde ser notada dentre as coortes mais recentes em relação às precedentes. Por sua vez, a idade possui relação côncava tanto com a fecundidade quanto com a participação.

Além disso, podem ser destacados os efeitos de outras variáveis de controle incluídas relacionadas às características pessoais, condição na família e local de residência. Como o fato de ser casada, que reduz a participação delas, além das diferenças regionais.

Outro resultado interessante encontrado foi o efeito dos salários ao longo da distribuição da renda domiciliar. Na equação de fecundidade, os efeitos dos salários são maiores nos décimos inferiores, refletindo o maior custo de oportunidade de ter filhos para as mulheres na cauda inferior da distribuição da renda. Na equação de participação no mercado de trabalho, a sensibilidade em relação aos salários é menor entre as mais pobres, o que pode indicar que outras variáveis estão afetando o comportamento das mulheres, tais como cultura, religião, falta de acesso a creches e à informação. Contudo, esses fatores não observados merecem maior atenção em trabalhos futuros e dos gestores públicos.

\section{Referências}

AVELINO, R.; MENEZES-FILHO, N. Estimação da oferta de trabalho das mulheres no Brasil. Estudos Econômicos, v. 33, n. 4, 2003.

BANCO MUNDIAL. Relatório sobre o Desenvolvimento Mundial de 2012, visão geral: Igualdade de gênero e desenvolvimento. Washington: Banco Internacional de Reconstrução e Desenvolvimento e Banco Mundial, 2011.

BECKER, G. An economic analysis of fertility. In: BECKER, G. Demographic and economic change in development countries. NBER, 1960. p. 209-231.

BECKER, G. A theory of the allocation of time. The Economic Journal, v. 75, n. 299, p. 493-517, Sept. 1965.

BERQUÓ, E.; CAVENAGHI, E. Fertilidade em declínio. Novos Estudos, n. 74, p. 11-15, Mar. 2006.

BRITO, F. A transição demográfica no contexto internacional. Textos para discussão, 317. Belo Horizonte: Cedeplar, 2007.

BRITO, F. A transição demográfica no Brasil: as possibilidades e os desafios para a economia e a sociedade. Textos para discussão, 318. Belo Horizonte: Cedeplar, 2007a.

BRITO, F. Transição demográfica e desigualdades sociais no Brasil. Revista Brasileira de Estudos da População, v. 25, n. 1, p. 5-26, jan./jun. 2008.

BROWNING, M. Children and Household Economic Behavior. Journal of Economic Literature, 
v. 30, n. 3, Sep., 1992, pp. 1434-1475.

BRUSCHINI, C.; LOMBARDI, M. R. O trabalho da mulher brasileira nos primeiros anos da década de noventa. In: ENCONTRO NACIONAL DE ESTUDOS POPULACIONAIS, 10., 1996, São Paulo. Anais...São Paulo: Abep, 1996, p.483-516.

BRUSCHINI, C. Gênero e trabalho no Brasil: Novas conquistas ou persistência da discriminação? (Brasil, 1985/1995). In: BALTAR DA ROCHA, M. I. (Org.). Trabalho e gênero: Mudanças, permanências e desafios. São Paulo: Editora 34, 2000.

CARVALHO, J. A. M.; BRITO, F. A demografia brasileira e o declínio da fecundidade no Brasil: Contribuições, equívocos e silêncios. Revista Brasileira de Estudos da População, v. 22, n. 2, p. 351-369, jul./dez. 2005.

CORSEUIL, C. H.; FOGUEL, M. N. Uma sugestão de deflatores para rendas obtidas a partir de algumas pesquisas domiciliares do IBGE. Texto para Discussão n.897. Rio de Janeiro: IPEA, jul. 2002.

D'ADDIO, A. C.; D'ERCOLE, M. M. Trends and determinants of fertility rates in OECD countries: The role of policies. Employment and migration working papers, n. 27. Franca: OECD, 2005, 91p.

DEL BOCA, D.; LOCATELLI, M. The determinants of motherhood and work status: A survey. DP n. 2414. Institute for the study of labor (IZA), Oct., 2006, 30p.

DI TOMMASO, M. L. A trivariate model of participation, fertility and wages: The Italian case. Cambridge Journal of Economics, v. 23, p. 623-640, 1999.

DI TOMMASO, M. L.; WEEKS, M. Decision Structures and Discrete Choices: An application to Labour Market Participation and Fertility. Cambridge Working Papers in Economics 0009. Faculty of Economics, University of Cambridge, 2000.

FONTOURA, N. O.; GONZALEZ, R. Aumento da participação de mulheres no mercado de trabalho: mudança ou reprodução da desigualdade? Mercado de trabalho, n. 41, p. 21-26, nov. 2009.

GREENE, W. H. Econometric analysis. 7. ed. Boston: Pearson, 2012.

GREENE, W. H. Marginal effects in the bivariate probit model. Department of Economics Working Paper Series, n. EC-96-11. New York University, June, 1996.

IBGE (Instituto Brasileiro de Geografia e Estatística). Censo Demográfico 2000. Nupcialidade e fecundidade. Resultados da Amostra. IBGE, Rio de Janeiro, 2003. 207 p.

IBGE (Instituto Brasileiro de Geografia e Estatística). Censo Demográfico 2010. Resultados gerais da amostra. IBGE, Rio de Janeiro, 2012. 65 p.

IBGE (Instituto Brasileiro de Geografia e Estatística). Projeção da População do Brasil por sexo e idade: 2000-2060. Rio de Janeiro, 2013. 21p.

LEONI, E. T. Renda familiar e trabalho da mulher na Região Metropolitana de São Paulo nos anos 80 e 90. In: BALTAR DA ROCHA, M. I. (Org.). Trabalho e gênero: Mudanças, permanências e desafios. São Paulo: Editora 34, 2000. p. 85-110.

MINCER, J. Market price, opportunity costs, and income effects. In: CHRIST, C. F. et al. Measurement in economics, studies in mathematical economics and econometrics in memory of Yehuda Grunfeld. Stanford, CA: Stanford University Press, 1963, p. 67-82. 
MINCER, J. The human capital earnings function. Schooling, experience and earnings. National Bureau of Economic Research, cap. V, p. 83-93, 1974.

MOFFIT, R. Profiles of Fertility, Labour Supply and Wages of Married Women: A Complete Life-Cycle Model. The Review of Economic Studies, v. 51, n. 2, Apr., pp. 263-278, 1984.

NEUMARK, D.; WASCHER, W. L. Mininum wages. Cambridge: MIT, 2008.

OECD (Organization Economic for Co-Operation and Development). Labour Force Statistics, 2007.

OLIVEIRA, P. R.; SCORZAFAVE, L. G.; PAZELLO, E. T. Desemprego e inatividade nas metrópoles brasileiras: As diferenças entre homens e mulheres. Nova Economia, Belo Horizonte, v. 19, n. 2, p. 291-324, maio/ago., 2009.

PAZELLO, E. T.; FERNANDES, R. A maternidade e a mulher no mercado de trabalho: Diferença de comportamento entre mulheres que têm e mulheres que não têm filhos. In: ENCONTRO DA ASSOCIAÇÃO NACIONAL DE PÓS-GRADUAÇÃO EM ECONOMIA, 31., 2004, João Pessoa. Anais... João Pessoa: ANPEC, 2004.

RAMOS, L. SOARES, A. L. Participação da mulher na força de trabalho e pobreza no Brasil. Revista de Economia Política, v. 15, n. 3 (59), p. 84-96, jul.-set. 1995.

SCORZAFAVE, L.; MENEZES-FILHO, N. Participação feminina no mercado de trabalho brasileiro: Evolução e determinantes. Pesquisa e Planejamento Econômico, v. 31, n. 3, p. 441-478, 2001.

SEDLACEK, G.; SANTOS, E. A mulher cônjuge no mercado de trabalho como estratégia de geração da renda familiar. Pesquisa e Planejamento Econômico, v. 21, n. 3, p. 449-470, 1991.

SOARES, S.; IZAKI, R. S. A participação feminina no mercado de trabalho. Texto para discussão, n. 923. Rio de Janeiro: IPEA, 2002, 22p.

TAVARES, P. A. Efeito do programa Bolsa-Família sobre a oferta de trabalho das mães. Economia e Sociedade, v. 19, n. 3 (40), p. 613-635, dez. 2010.

WAJNMAN, S.; RIOS-NETO, E. Quantas serão as mulheres: cenários para a atividade feminina. In: ROCHA, M. I. B. (Coord.). Trabalho e gênero: mudanças, permanências e desafios. ABEP, NEPO/UNICAMP e CEDEPLAR/UFMG, Editora 34, p. 59-84, 2000.

WILLIS, R. A new approach to the economic theory of fertility behavior. Journal of Political Economy, v. 82, S14-S64, 1973.

\section{Sobre os autores}

Marina Silva Cunha-mscunha@uem.br

Departamento de Economia, Universidade Estadual de Maringá, Maringá, PR.

MarcosRobertoVasconcelos -mrvasconcelos@uem.br

Departamento de Economia, Universidade Estadual de Maringá, Maringá, PR.

\section{Sobre $\mathrm{o}$ artigo}

Recebido em 11 de março de 2014. Aprovado em 16 de fevereiro de 2015. 


\section{APÊNDICE}

Tabela A1 Efeitos marginais para as equações de fecundidade e de participação, Brasil, $1995-2009$ a

\begin{tabular}{lrr}
\hline Variável & Fecundidade & Participação \\
\hline Salário estimado & $-0,15258^{* *}$ & $0,22680^{* *}$ \\
\hline Idade & $0,16410^{* *}$ & $0,02944^{* *}$ \\
\hline Idade ao quadrado & $-0,00235^{* *}$ & $-0,00049^{* *}$ \\
\hline Rendimento domiciliar & $-0,00001^{* *}$ & $2,4 \mathrm{E}-06^{* *}$ \\
\hline Branca & $-0,02643^{* *}$ & $-0,04154^{* *}$ \\
\hline Chefe & $0,31201^{* *}$ & $0,10240^{* *}$ \\
\hline Casadas & $0,28916^{* *}$ & $-0,10590^{* *}$ \\
\hline Nordeste & $-0,04632^{* *}$ & $0,00796^{* *}$ \\
\hline Sudeste & $-0,06469^{* *}$ & $0,05419^{* *}$ \\
\hline Sul & $-0,03365^{* *}$ & $0,09838^{* *}$ \\
\hline Centro-Oeste & $-0,05135^{* *}$ & $0,04037^{* *}$ \\
\hline Área urbana & $-0,04677^{* *}$ & $0,03361^{* *}$ \\
\hline Região metropolitana & $-0,02352^{* *}$ & $0,00218^{* *}$ \\
\hline
\end{tabular}

Fonte: Dados da pesquisa.

*Valores estatisticamente significativos com nível de 5\%.

* Valores estatisticamente significativos com nível de 1\%.

${ }^{a}$ As estimativas incluíram como controle as variáveis binárias que indicam as coortes e os anos da pesquisa. 
Tabela A2 Efeitos marginais obtidos com base nas probabilidades bivariadas, Brasil, $1995-2009$

\begin{tabular}{lrrrrr}
\hline Variável & $\mathbf{( F} \cap \mathbf{P})$ & $\mathbf{( N F} \cap \mathbf{P})$ & $\mathbf{( F} \cap \mathbf{N P})$ & $\mathbf{( N F} \cap \mathbf{N P})$ \\
\hline Salário estimado & $0,01558^{* *}$ & $0,21122^{* *}$ & $-0,05864^{* *}$ & $-0,16816^{* *}$ \\
\hline Idade & $0,11598^{* *}$ & $-0,08654^{* *}$ & $-0,07755^{* *}$ & $0,04811^{* *}$ \\
\hline Idade ao quadrado & $-0,00170^{* *}$ & $0,00120^{* *}$ & $0,00115^{* *}$ & $-0,00065^{* *}$ \\
\hline Rendimento domiciliar & $-0,00001^{* *}$ & $0,00001^{* *}$ & $2,7 \mathrm{E}-06^{* *}$ & $-0,00001^{* *}$ \\
\hline Branca & $-0,03668^{* *}$ & $-0,00486^{* *}$ & $0,03130^{* *}$ & $0,01024^{* *}$ \\
\hline Chefe & $0,26314^{* *}$ & $-0,16074^{* *}$ & $-0,15127^{* *}$ & $0,04888^{* *}$ \\
\hline Casadas & $0,13829^{* *}$ & $-0,24419^{* *}$ & $-0,04497^{* *}$ & $0,15088^{* *}$ \\
\hline Nordeste & $-0,02495^{* *}$ & $0,03292^{* *}$ & $0,01340^{* *}$ & $-0,02136^{* *}$ \\
\hline Sudeste & $-0,01395^{* *}$ & $0,06814^{* *}$ & $-0,00344^{* *}$ & $-0,05075^{* *}$ \\
\hline Sul & $0,02509^{* *}$ & $0,07330^{* *}$ & $-0,03965^{* *}$ & $-0,05874^{* *}$ \\
\hline Centro-Oeste & $-0,01377^{* *}$ & $0,05414^{* *}$ & $-0,00279^{* *}$ & $-0,03758^{* *}$ \\
\hline Área urbana & $-0,01156^{* *}$ & $0,04517^{* *}$ & $0,00161^{* *}$ & $-0,03522^{* *}$ \\
\hline Região metropolitana & $-0,01352^{* *}$ & $0,01571^{* *}$ & $0,00782^{* *}$ & $-0,01000^{* *}$ \\
\hline
\end{tabular}

Fonte: Dados da pesquisa.

*Valores estatisticamente significativos com nível de 5\%.

**Valores estatisticamente significativos com nível de 1\%.

${ }^{a}$ As estimativas incluíram como controle as variáveis binárias que indicam as coortes e os anos da pesquisa. 